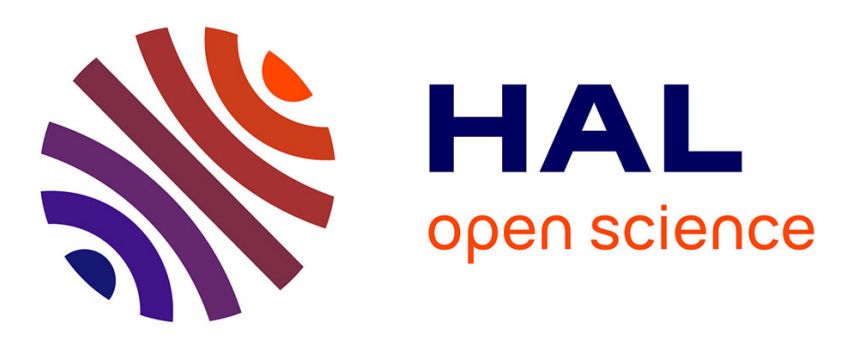

\title{
Three multispectral configurations of a snapshot kaleidoscope-based camera in long wavelength infrared spectral band
}

Adrien Mas, Guillaume Druart, Patrick Bouchon, Grégory Vincent, Sylvain Favier, Eric Compain, Nicolas Guérineau

\section{To cite this version:}

Adrien Mas, Guillaume Druart, Patrick Bouchon, Grégory Vincent, Sylvain Favier, et al.. Three multispectral configurations of a snapshot kaleidoscope-based camera in long wavelength infrared spectral band. Applied optics, 2020, 59 (26), pp.7779-7791. 10.1364/AO.399873 . hal-03104228

\section{HAL Id: hal-03104228 \\ https://hal.science/hal-03104228}

Submitted on 22 Feb 2021

HAL is a multi-disciplinary open access archive for the deposit and dissemination of scientific research documents, whether they are published or not. The documents may come from teaching and research institutions in France or abroad, or from public or private research centers.
L'archive ouverte pluridisciplinaire HAL, est destinée au dépôt et à la diffusion de documents scientifiques de niveau recherche, publiés ou non, émanant des établissements d'enseignement et de recherche français ou étrangers, des laboratoires publics ou privés. 


\title{
Three multispectral configurations of a snapshot kaleidoscope-based camera in long wavelength infrared spectral band
}

\author{
Adrien Mas, ${ }^{1,2, *}$ Guillaume Druart, ${ }^{1}$ Patrick Bouchon, ${ }^{1}$ (D) GrÉgory Vincent, ${ }^{1}$ \\ Sylvain Favier, ${ }^{2}$ Eric Compain, ${ }^{2}$ and Nicolas GuÉrineau ${ }^{1}$ \\ ${ }^{1}$ Département Optique et Techniques Associées, ONERA, Université Paris Saclay F-91123 Palaiseau, France \\ ${ }^{2}$ Bertin Technologies, 155 Rue Louis Armand ZI, 13290 Aix-en-Provence, France \\ *Corresponding author: adrien.mas@onera.fr
}

Received XX Month XXXX; revised XX Month, XXXX; accepted XX Month XXXX; posted XX Month XXXX (Doc. ID XXXXX); published XX Month $X X X X$

\begin{abstract}
In the field of spectral imaging, numerous instruments use scanning-based technologies. However, the temporal dimension of these systems, whether to scan the spectrum or scan the scene, can be an issue for some applications. This is particularly the case when trying to observe and identify rapid temporal variations in a fixed scene or detecting objects of interest when moving. In this case, it is suitable to observe the desired spectral information of the scene simultaneously, and so-called snapshot systems have been thus investigated. In this paper, we study the ability of a kaleidoscope-based multiview camera to acquire multispectral information in the long wavelength infrared. Several strategies and technologies will be compared to add the spectral function inside the different blocks of a kaleidoscope-based camera: the front lens, the kaleidoscope, or the reimaging lens. The studied camera uses an uncooled infrared detector and thus must deal with the issue of having a large aperture. $\odot 2020$ Optical Society of America
\end{abstract}

https://doi.org/10.1364/XXXXX

\section{INTRODUCTION}

Multispectral imaging systems sample the spectral irradiance of a scene and thus collect a three-dimensional (3D) data set typically called a data cube. Since data cubes are of a higher dimensionality than the two-dimensional (2D) detector arrays, system designers must resort to either measuring time-sequential 2D slices of the cube (scanning system) or simultaneously measuring all elements of the data cube by dividing it into multiple 2D elements (snapshot system) that can be recombined into a $3 \mathrm{D}$ data cube in postprocessing.

In the field of spectral imaging, a large proportion of instruments use scanning-based technologies [1]. Among the scanning systems are the "point scanning spectrometers" in which the sampling points of a scene are scanned and spectrally spread along a line of the detector. In pushbroom spectrometers, the observed scene is a $1 \mathrm{D}$ line that is scattered over a $2 \mathrm{D}$ detector. To temporally acquire a spectrum, a tunable filter camera can be used too. The tunable filter can be, for example, a filter wheel [2] or a mechanically tuned Fabry-Perot etalon [3,4], a liquid-crystal tunable filter (LCTF) [5] or an acousto-optic tunable filter (AOTF) [6]. Other technologies, such as the imaging Fourier transform spectrometer, scan one mirror of a Michelson interferometer in order to obtain measurements at multiple optical path difference (OPD) values-the Fourier domain being equivalent to a tunable filter camera $[7,8]$.

However, the temporal dimension of these systems, whether to scan the spectrum or scan the scene, can be problematic for some applications. This is particularly the case when trying to observe and identify rapid temporal variations in a fixed scene, such as gas plumes. Their quick evolution makes the coregistration of the spectral content of different images difficult with scanning-based technologies, leading to errors of detection and identification as well as false alarms. Snapshot spectral cameras are thus best suited for this kind of application. For instance, an array of low-cost uncooled infrared cameras has been studied for gas detection [9]. The concept of multispectral beam-splitting cameras could be evaluated too. The idea of using multiple beam splitters, such as separator cubes, for color imaging has been explored for some time [10-12]. However, in the infrared, uncooled thermal detectors such as microbolometers produce a signal that can easily drift with the environmental temperature. Snapshot spectral cameras using a single focal plane array (FPA) are often suitable because the spectral images drift in the same way, and radiometric comparison can be thus considered.

Numerous types of snapshot multispectral architectures using a single FPA have already been studied, but they show limitation when using an uncooled thermal detector that 
requires low-aperture systems. Spectrally resolving detector arrays concepts could be thus considered [13], but this technology is not enough mature in the long wavelength infrared (LWIR) yet. Another concept, the integration field spectrometry (IFS), divides the field of view into several field sampling points by one of the following techniques: with mirrors (IFSMs) [14], lens arrays (IFS-Ls) [15], or optical fiber bundles (IFS-Fs) [16]. Then each sampling point is spectrally dispersed on the FPA. However, these architectures tend to strongly limit spatial resolution in favor of spectral resolution. For a gas detection and identification application in the infrared bandwidth, a limited number of spectral bands can be sufficient. Moreover, IFS spreads the spectral content on several pixels, so each pixel acts like a bandpass filter. When using uncooled thermal detectors, a set of bandpass and longpass filters is usually preferred for sensibility issues [17]. Finally, another concept is the multiaperture filtered camera, which uses a monolithic lenslet array, also called TOMBO (thin observation module by bound optics) system [18]. By associating a different filter with each optical channel, the camera becomes easily multispectral. An LWIR uncooled TOMBO camera has already been made [19]. However, its major limitation relies on the relationship between the $F$-number $(F \#)$ and the field of view to avoid either blind area on the FPA or field overlap between adjacent channels [20]. As a result, a narrow field of view with a high numerical aperture induces unused pixel areas on the FPA [19,20]. To overcome this constraint, Carles's team proposed a two-stage freeform TOMBO architecture allowing an increase of the numerical aperture by using off-axis optics to tilt the optical axis of each channel [21]. However, freeform surfaces that can be different from one channel to another increase the complexity of the optical system drastically. Since unconventional lenses have to be made and aligned, this solution becomes difficult to apply for more than nine images. Finally, multichannel architectures must manage the cross talk between the different channels, usually by using walls, since a field stop is not easily implemented for a high numerical aperture [22].

Light-field type cameras were studied too [23]. Among these different concepts, the single-aperture light-field design based on a kaleidoscope does not suffer from the limitations of multiaperture systems [24]. In a previous article [25], we showed that an LWIR kaleidoscope-based multiview system can be designed. In terms of size, this system is equivalent to a lenslet design with a front afocal [26], and it has the following advantages: it can answer the high numerical aperture challenge required by microbolometers while having a great flexibility with the field of view and while limiting the unused pixels between the subimages. It only uses conventional components, thus enabling rapid and cost-effective manufacturing and assembling. It also has the advantage of being modular, and the field of view can be adapted by only changing the front lens. References [24,27] show that a multispectral kaleidoscope-based camera was possible, but at the cost of increasing the complexity and the size of the design, i.e., by adding a reimaging stage to the original architecture.

In this paper, we propose several methods to add the multispectral function to the kaleidoscope architecture without adding this optical stage. Each design is analyzed in terms of optical performance, technical complexity, and technological maturity. In Section 2, we recall the different blocks of a kaleidoscope-based system, and then in the following parts, we investigate the possibility of adding a multispectral function in each block: the reimaging lens, the kaleidoscope, and the front lens.

\section{RECALL OF THE DESIGN PRINCIPLE OF A KALEIDOSCOPE-BASED SYSTEM}

The principle of a three-stage kaleidoscope-based system is to place a kaleidoscope, which is a tube made of four plane mirrors, between a first imaging lens and a reimaging one. An intermediate image is formed by the front lens at the entrance of the kaleidoscope. By reflection in the kaleidoscope, the cones of incident beams are divided into $N \times N$ cones with different orientations that form $N \times N$ virtual copies of the image produced by the front lens in the intermediate image plane. This array of images is then focused by the reimaging lens on the FPA. This architecture is illustrated in Fig. 1, which is adapted from Ref. [25].

To add the multispectral function to this multiview architecture, Irhke's team adds a second reimaging stage after a second intermediate image plane, as shown in Fig. 2 [24]. A filter array is positioned in this second intermediate plane, and each filter is associated with a subimage. These multispectral subimages are then reimaged on the FPA by the second reimaging lens.

This architecture makes it easy to change the filter array and therefore allows flexibility of use. However, this configuration increases the footprint of the camera, its assembly, and the difficulty of its athermalization. So, for industrialization concerns, the multispectral function should rather be implemented in the three-stage design illustrated in Fig. 1. In Fig. 1, the rays related

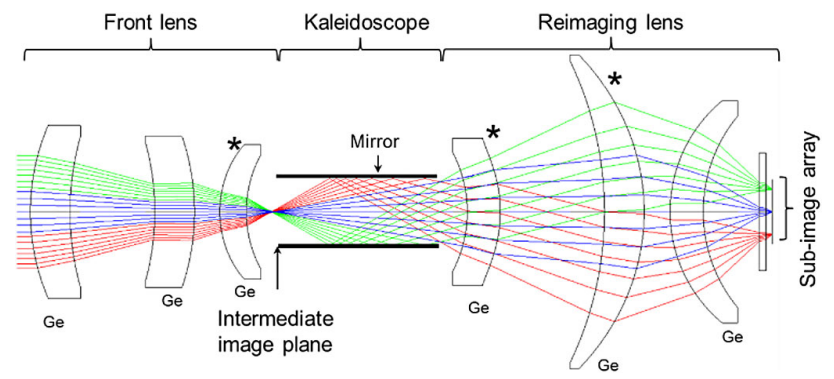

Fig. 1. Multiview design based on a kaleidoscope. Figure adapted from Ref. [25]. Surfaces marked with * are aspheric.

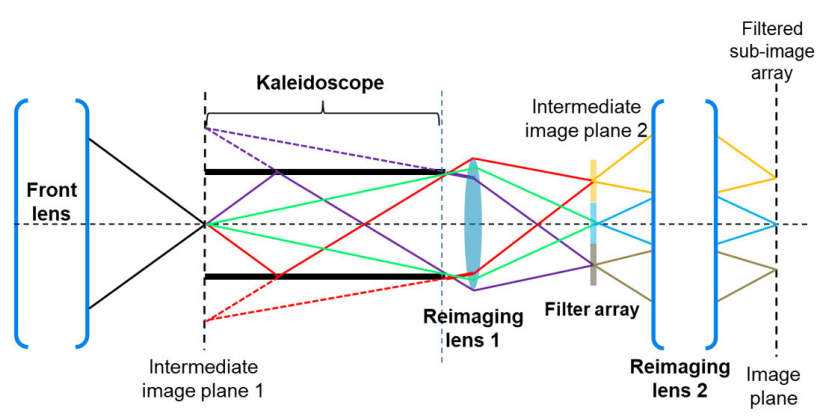

Fig. 2. Multispectral kaleidoscope-based architecture using a second reimaging lens. A filter array is placed in a second intermediate image plane. 
to the central image points of each subimage are drawn with a different color. Where the rays of different colors are well separated in space (no overlap), a set of different filters can be placed and each subimage can be differently filtered. So, the multispectral function can be implemented as close as possible to the FPA. As the mirrors are the key elements to create the array of subimages, the multispectral function can be integrated on the mirrors by replacing them with reflective filters as well. Finally, the light-field property of the kaleidoscope-based architecture divides the entrance pupil of the front lens into subpupils, each being associated with a subimage. As a result, the array of filters could be placed at a physical entrance pupil of the front lens or at a stop diaphragm inside the front lens.

\section{PLACING THE FILTER ARRAY NEAR THE FPA}

A first straightforward solution to integrate the multispectral function into a three-stage kaleidoscope architecture is to position a filter array as close as possible to the FPA, each filter being associated with a subimage and having the same dimensions. However, the packaging of a microbolometer detector must be considered. Indeed, in order to place the microbolometers under vacuum, this detector has a sealing window, which limits the positioning of the filter array. This distance between the filter array and the focal plane may induce an overlap of some rays of the incident beam on adjacent filters, as illustrated in Fig. 3. Indeed, we clearly see that the incident beams retropropagated from image points at the border of the subimages can overlay several filters. This overlap on several filters will result in a spectral cross talk at the border of the subimages.

This cross talk can be quantified according to the size of the subimages, the distance between the filters and the FPA, the thickness of the window, and the $F \#$ of the system. If we assume that the system is telecentric for the central image, we can establish formulas to evaluate the spectral cross talk by analyzing the retropropagation of the incident beams from each image point of the central image to the filter array and by quantifying the surface area of the intersection of the cone beam with the central filter. The calculation is developed in Appendix A. Figure 4 shows a simulation of the percentage of correctly filtered rays for each point in the central image made with the following values: $1 \mathrm{~mm}$ of air distance between the filter and the FPA,

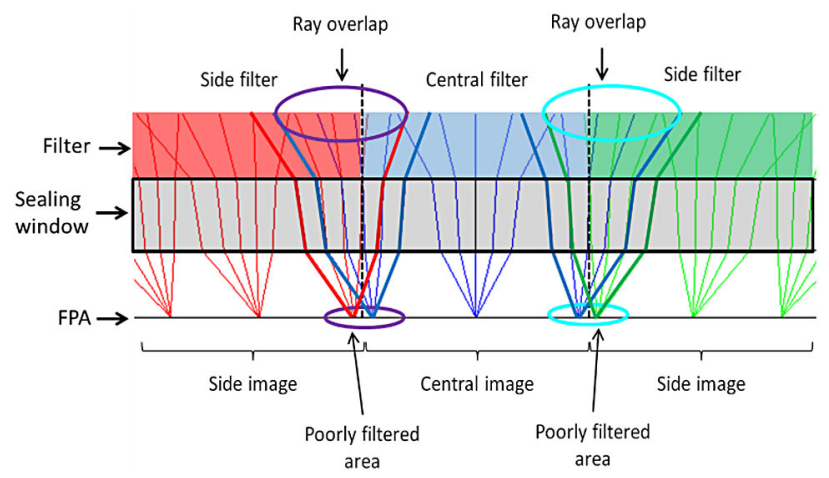

Fig. 3. Spectral cross-talk phenomenon due to a sealing window between the FPA and the array of filters. The incident beams retropropagated from image points at the border of the subimages can overlay several filters.
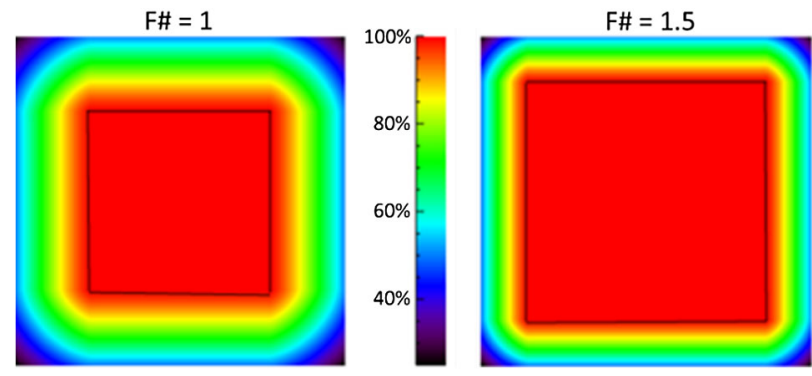

Fig. 4. Simulation of the percentage of correctly filtered rays for each point in the central image with the assumptions that air distance between the filter and the FPA is equal to $1 \mathrm{~mm}$, and the window is in germanium and has a thickness of $1 \mathrm{~mm}$. The perfectly filtered area is framed in black. On the left is the simulation for a $F \#$ of 1 , on the right, for a $F \#$ of 1.5 .

$1 \mathrm{~mm}$ of germanium window (with a refractive index of 4 ), a size of subimage of $3 \mathrm{~mm}$ and an $F \#$ of 1 or 1.5 (an $F \#$ of 1.5 is usually the maximal accepted $F \#$ for an optical system using an uncooled infrared microbolometer).

In Fig. 4, the area framed in black is the area of the subimage for which all rays have been correctly filtered by the matching filter of the central image. At the corners of the subimage, the percentage of correctly filtered rays drops to $25 \%$ because the incident beam is equally filtered by the four adjacent filters. For an $f / 1$ aperture, the percentage of the subimage that is perfectly filtered is $29 \%$. It is $51 \%$ for an $f / 1.5$ system.

The spectral overlap creates a lot of unused areas on the FPA for our experimental camera, and we lost one advantage of this system over other multispectral solutions [26]. We can conclude that the distance between the focal plane and the filters is critical in the case of a high opened system, typically $f / 1$. We hope the development of compact packaging of microbolometer detectors can save this solution of implementing the multispectral function on the focal plane. For example, the wafer-level packaging (WLP) technology, as presented in the FLIR Systems patent [28], can provide a solution by reducing the distance between the filters and the focal plane to a few hundred micrometers. With such a detector, the distance between filters and FPA could be less than $600 \mu \mathrm{m}$ of air thickness and $500 \mu \mathrm{m}$ of thickness for a germanium window. With these hypothetical values, the simulation was done for apertures of $f / 1$ and $f / 1.5$. The results are illustrated in Fig. 5 . With an $f / 1$ aperture, $58 \%$ of the image
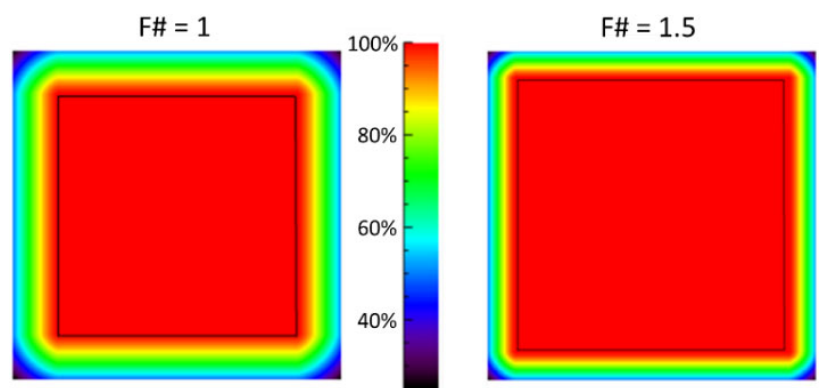

Fig. 5. Simulation of the percentage of correctly filtered rays for any point in the central image with WLP technology. The perfectly filtered area is framed in black. On the left is the simulation for a $F \#$ of 1 , on the right, for a $F \#$ of 1.5 . 

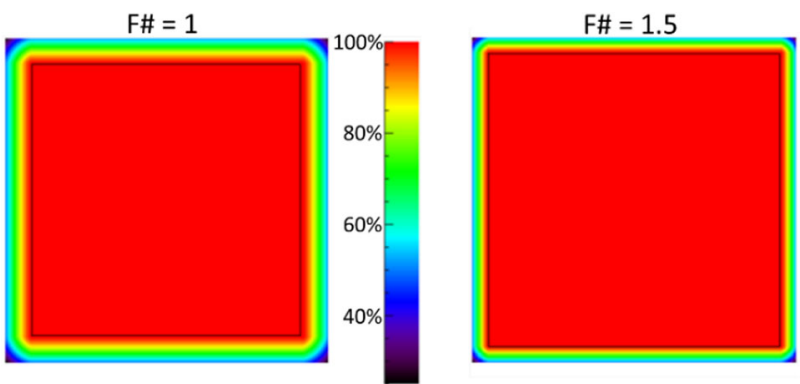

Fig. 6. Simulation of the percentage of correctly filtered rays for any point in the central image with PLP technology. The perfectly filtered area is framed in black. On the left is the simulation for a $F \#$ of 1 , on the right, for a $F \#$ of 1.5 .

would be perfectly filtered, and with an $f / 1.5$ aperture, $77 \%$ of the image would be perfectly filtered, which would be much more relevant for a kaleidoscope-based multispectral infrared system.

An even more compact technology called pixel-level packaging (PLP) could further increase the performance of such architecture [29,30]. Simulations were done with $300 \mu \mathrm{m}$ of air distance and $100 \mu \mathrm{m}$ of germanium window; the results are illustrated in Fig. 6. In this case, 85\% of the subimage is perfectly filtered for a system opened at $f / 1$ and more than $90 \%$ of the subimage is perfectly filtered for a system opened at $f / 1.5$.

We can therefore conclude that the implementation of the multispectral function on the FPA depends on the technological evolution of uncooled LWIR detectors that move toward compact packaging solutions.

\section{PLACING THE FILTERS ON THE KALEIDOSCOPE'S MIRRORS}

The kaleidoscope's mirrors are the key element of this multiview system. The rays producing the central subimage cross through the kaleidoscope without any reflections on the mirrors, whereas the rays producing the side images are reflected by one of the four mirrors, and the rays producing the diagonal images are reflected by two adjacent mirrors. So, placing the filters on the mirrors could be an easy way to get the kaleidoscope-based system multispectral. Moreover, placing the filters on the mirrors prevents any spectral cross talk between the subimages, as observed in the previous section. This solution preserves a threestage kaleidoscope, but the number of spectral bands is limited by the number of mirrors equal to four. Since the central image is not spectrally filtered, an equivalent to the set of high-pass and low-pass filters commonly used with microbolometers could be a set of notch filters implemented on the mirrors, as illustrated in Fig. 7. These filters are characterized by a central wavelength where the absorption of light is maximal. From either side of this wavelength, the reflection is improved. In this configuration, since the diagonal images are made by rays reflecting on two adjacent mirrors, they will be filtered by two notch filters and will be thus a spectral mixing of two lateral images.

Such notch filters could be produced by common multilayer thin films. However, there are several technological barriers because there are few transparent materials at these wavelengths, and the thickness and adhesion of each layer are also critical

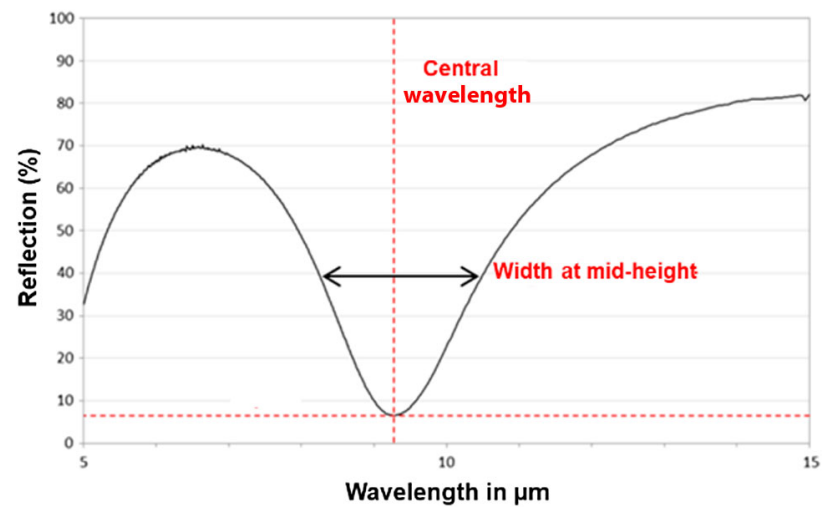

Fig. 7. Example of a reflective spectral band of a notch filter.

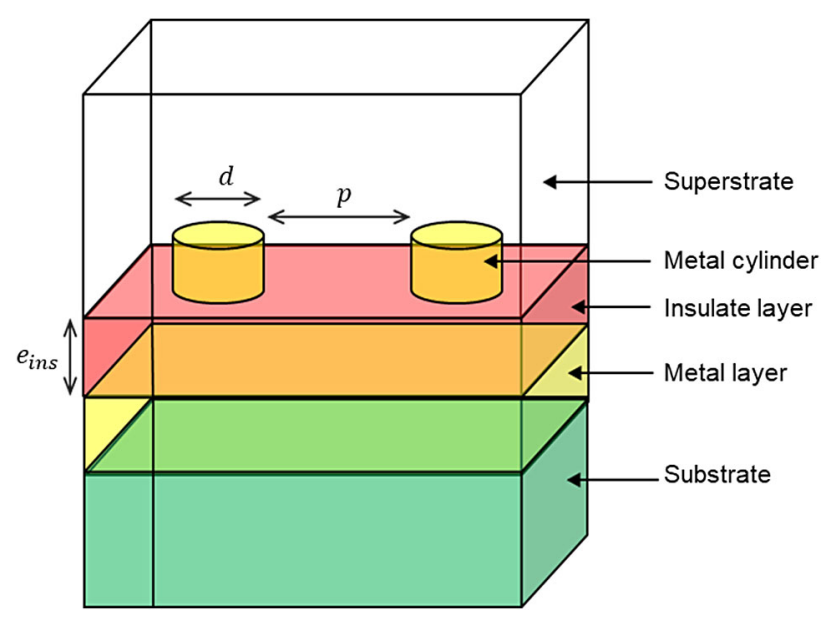

Fig. 8. Architecture of MIM-type plasmonic filter.

issues. Besides, each filter will need a different stack of layers, thus increasing the cost of production. An alternative is to consider plasmonic filters [31-35]. They consist in a repetition of metal-insulator-metal (MIM)-type patterns, detailed in Fig. 8 [36-38]. In the configuration presented in this figure, the superstrate is air, and the metal cylinders and the metal layer are made of chromium. The insulating layer, of thickness $e_{\text {ins }}$, and the substrate are made of silicon $(\mathrm{Si})$. The use of plasmonic filters can simplify the fabrication of filters compared to multilayer dielectric approaches because the reflective spectral band of the filters are set by the shape of the plasmonic patterns: the diameter of the cylinders $d$ and the period of the patterns $p$. Several filters with different reflective spectral bands can therefore be made on the same wafer, and this approach is also a way to reduce cost.

Notch filters with plasmonic patterns have been fabricated to replace the mirrors in our kaleidoscope-based camera. The period of the plasmonic structures is $2400 \mathrm{~nm}$. The diameter of the metal cylinders is $1200 \mathrm{~nm}$. The thickness of the chromium layer is $110 \mathrm{~nm}$ and that of the Si layer is $270 \mathrm{~nm}$. Their reflective spectra have been measured for different angles of incidence with a Fourier transform infrared spectrometer (Bruker Vertex 70v), as shown in Fig. 9.

Figure 9 shows that the shape of the reflective spectral band degrades at high angles of incidence. The value of the central wavelength decreases when the angle of incidence increases, as 


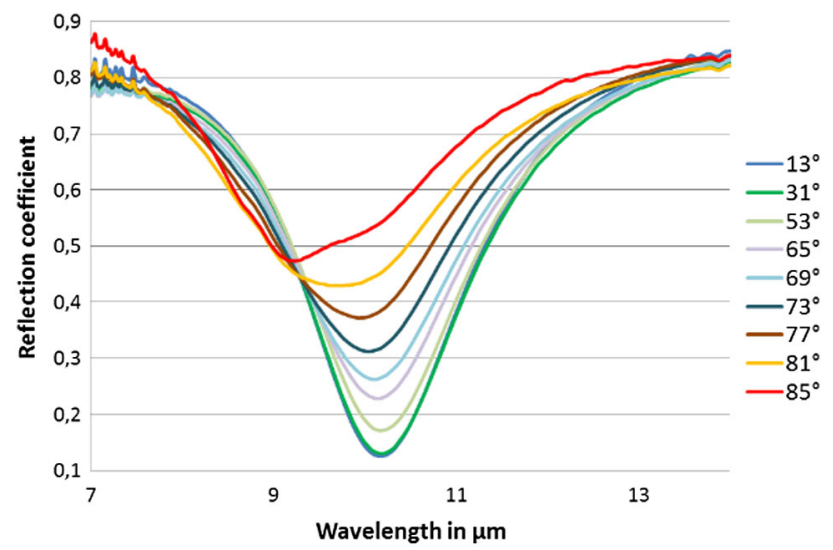

Fig. 9. Reflective spectral band of a plasmonic notch filter for several angles of incidence of the light on the mirror (the angles of incidence are given to the normal of the mirror).

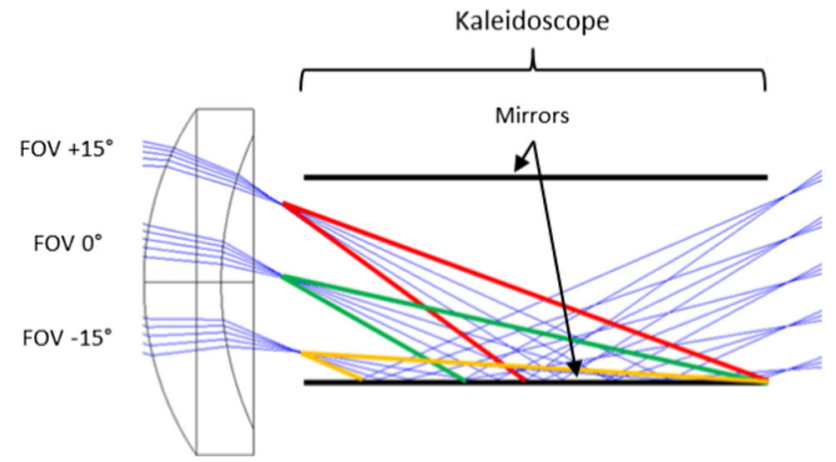

Fig. 10. Focus on the kaleidoscope part where the cones of rays reflecting on a mirror of three field points are drawn (in red for the field point of $+15^{\circ}$, in green for the central field point, and in yellow for the field point of $-15^{\circ}$ ).

does the absorption value at the central wavelength. It could be considered that the reflective spectral band is no longer acceptable above the angle of incidence of $60^{\circ}$. Unfortunately, we evaluated the maximum angle of incidence of the ray that reflects on the kaleidoscope mirrors with ZEMAX software to $85^{\circ}$, a value much higher than $60^{\circ}$. Figure 10 shows a focus of the kaleidoscope part where the cone of rays from the border of the field of view of the camera $\left( \pm 15^{\circ}\right)$ and the cone of rays of the central field point reflect on a mirror. We can see that the average angle of incidence of the rays is not the same for the different field-of-view points. In the case of our demonstrator, the angles of rays from the field point $+15^{\circ}$ reflecting on the mirrors are between $50^{\circ}$ and $70^{\circ}$, whereas the cone angles of rays from the opposite field point $\left(-15^{\circ}\right)$ are between $70^{\circ}$ and $85^{\circ}$.

By considering the measurements of Fig. 9 for different angles of incidence and with the assumption that the light is equally distributed in all angles of incidence at first approximation, the reflective spectral band can be obtained by integration for different field points. The result is shown in Fig. 11. A deterioration of the spectral filtering is observed as a function of the field of view, and it can therefore be concluded that the important angles of incidence of the light rays on the mirrors cause both a degradation of the spectral filtering performance and

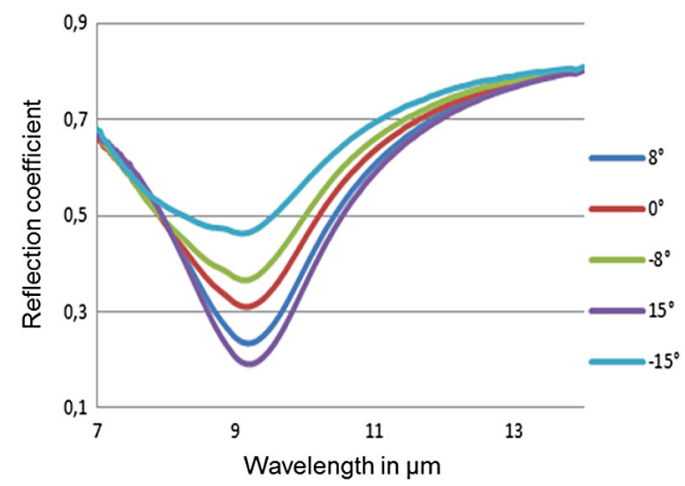

Fig. 11. Reflective spectral bands for different field points: $15^{\circ}, 8^{\circ}$, $0^{\circ},-8^{\circ},-15^{\circ}$.

a nonuniformity filtering effect over the field of view on each subimage.

In the following part of this section, we investigate two ways to correct the response of these plasmonic filters. In the first case, we study an asymmetric MIM configuration that is able to absorb light at high incidence wavelengths. In the second case, we keep cylindrical plasmonic structures, but we immerse them in a high refractive index material to decrease the incidence angle of the rays on the plasmonic structures. In the two cases, the computations are performed using the rigorous coupled wave analysis (RCWA) [39]. The plasmonic structure (cylindrical or rectangular) and the metal layer are in chromium, and its permittivity is modeled by a Drude model: $\varepsilon=\varepsilon_{\infty}\left(1-\frac{\omega_{p}^{2}}{\left(\omega^{2}+i \omega \omega_{\tau}\right)}\right)$, with $\varepsilon_{\infty}=1, \quad \omega_{p}=36650 \mathrm{~cm}^{-1}$, $\omega_{\tau}=440 \mathrm{~cm}^{-1}$, as described in [40].

The first solution aims at readapting the MIM resonator for higher incidence angles. For the sake of simplicity, the top patterned metallic layer consists in a rectangular patches array (length $L_{x}$, width $L_{y}$ ), the asymmetry of the pattern being used to counterbalance the difference of resonance behavior for each polarization at high incidence angles. Figure 12 illustrates the response of an optimized geometry with parameters $\left(L_{x}=1.2 \mu \mathrm{m}, L_{y}=1.05 \mu \mathrm{m}, e_{\text {ins }}=0.7 \mu \mathrm{m}, e_{\text {metal }}=0.2 \mu \mathrm{m}\right.$, and a $4 \mu \mathrm{m}$ period). The insulator is taken as amorphous $\mathrm{Si}$ $\left(n_{\text {Si: } a}=3.5\right)$. Both polarizations have a similar behavior, with a resonance at an $11.6 \mu \mathrm{m}$ wavelength and a reflectivity below $23 \%$ for a range of angles between $55^{\circ}$ and $85^{\circ}$. The quality factors of the resonances are slightly higher in the cases of the TM polarization. At a wavelength of $9 \mu \mathrm{m}$, there is a Fabry-Perot resonance in the amorphous $\mathrm{Si}$ layer; it could be avoided by replacing the amorphous $\mathrm{Si}$ by a lower refractive index material. Despite an improvement in reflectivity at the central wavelength for high incidence (compared to the Fig. 9), the spectral performance of the filter still varies greatly with the angle of incidence of the rays.

As the important angles of incidence of the light rays on the mirrors are identified as the limiting factor for a multispectral kaleidoscope-based system with filters on the mirrors, we propose to immerse the plasmonic structures in a material of high refractive index so that the refracted rays have a smaller angle of incidence on the plasmonic patterns, as illustrated in Fig. 13. 

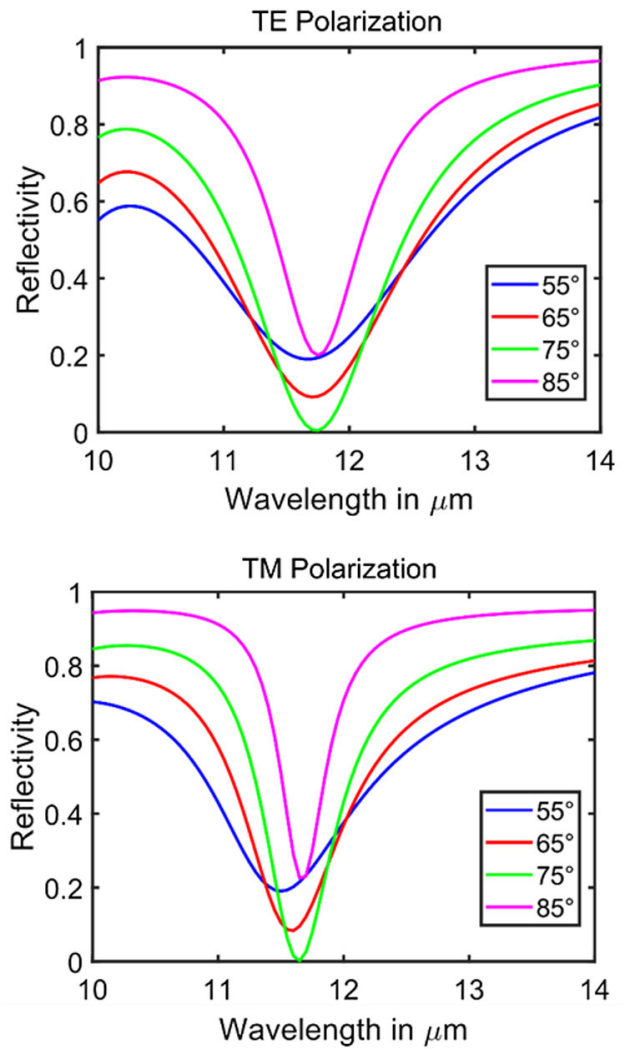

Fig. 12. Reflective spectral bands calculated in TE or TM polarization for several angles of incidence for an asymmetric MIM resonator.

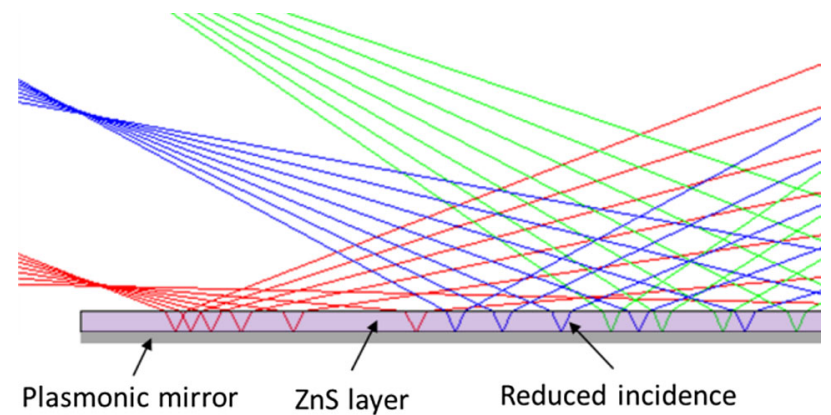

Fig. 13. Focus on a mirror of the kaleidoscope, where the light rays are first refracted through a substrate with a high index of refraction before reflecting on the plasmonic mirrors. Reduced angles of incidence on the mirrors are clearly seen in this figure.

The difference with the previous filters is the superstrate, which is made of a high-index material and the plasmonic structures, which are cylindrical. Two materials for the superstrate are considered: $\mathrm{Si}\left(n_{\mathrm{Si}}=3.3\right)$ and $\mathrm{ZnS}\left(n_{\mathrm{ZnS}}=2.2\right)$. In each case, the insulating layer is considered to be made of the same material as the superstrate to allow a better material compatibility, particularly in terms of mechanical stresses. However, this deprives us of a degree of freedom to optimize the design. We consider an infinite superstrate, i.e., by ignoring the air/superstrate interface (we consider that the interface can be treated with an antireflection coating). Moreover, for the simulations, the angle of incidence in the superstrate is not equal
Table 1. Equivalence between Incidence Angle in Air and Incidence Angle in the Si Superstrate

\begin{tabular}{lc}
$\begin{array}{l}\text { Angle of Incidence } \\
\text { in the Air }\end{array}$ & $\begin{array}{c}\text { Angle of Incidence in the Si } \\
\text { Superstrate }\end{array}$ \\
\hline $0^{\circ}$ & $0^{\circ}$ \\
$20^{\circ}$ & $5,94^{\circ}$ \\
$40^{\circ}$ & $11,23^{\circ}$ \\
$60^{\circ}$ & $15,21^{\circ}$ \\
$80^{\circ}$ & $17,36^{\circ}$ \\
$90^{\circ}$ & $17,63^{\circ}$ \\
\hline
\end{tabular}

to the angle of incidence in the air. Thus, equivalences are given by the refraction law, and the values for each material will be recalled. The metal thicknesses are always the same: $300 \mathrm{~nm}$ for the mirror and $110 \mathrm{~nm}$ for the height of the cylinders. The reflective spectral bands will be simulated for TE or TM polarization, respectively, when the electric or the magnetic field is orthogonal to the incidence plane.

The first straightforward material for the superstrate is the $\mathrm{Si}$, since many photolithography processes have been developed for this material. For the Si superstrate, we consider the following parameters:

$$
\begin{aligned}
n_{\mathrm{Si}} & =3,3, \\
p & =650 \mathrm{~nm}, \\
d & =500 \mathrm{~nm}, \\
e_{\text {ins }} & =50 \mathrm{~nm} .
\end{aligned}
$$

The equivalence between the angles of incidence in air and in the Si superstrate is indicated in Table 1. The reflective spectral bands for TE or TM polarization and for different angles of incidence are given in Fig. 14 . The central wavelength has a reflection coefficient of $10 \%$ for angles of incidence from $0^{\circ}$ to $18^{\circ}$ in the Si superstrate, and the shapes of the reflective spectral bands are the same for all the angles of incidence. This simulation shows that we can obtain an efficient and uniform spectral filtering for all the side subimages when immersing the plasmonic patterns in a superstrate with a high index of refraction. However, geometries of the plasmonic patterns are very small and require important lithography resources, such as electronic lithography or nanoimprint. Moreover, the maximum reflection coefficient is lower than $75 \%$; this may be improved with different materials for the metal layer, the metal cylinder, or the substrate. Finally, Si can have absorption spectral bands in the LWIR bandwidth. Studying other materials for the superstrate could be useful.

For example, $\mathrm{ZnS}$ is a common material for LWIR optics. For the $\mathrm{ZnS}$ superstrate, we consider the following parameters:

$$
\begin{aligned}
n_{\mathrm{ZnS}} & =2,2, \\
p & =1350 \mathrm{~nm}, \\
d & =750 \mathrm{~nm}, \\
e_{\text {ins }} & =90 \mathrm{~nm} .
\end{aligned}
$$

The equivalence between the angles of incidence in air and in a superstrate made of $\mathrm{ZnS}$ is indicated in Table 2. 

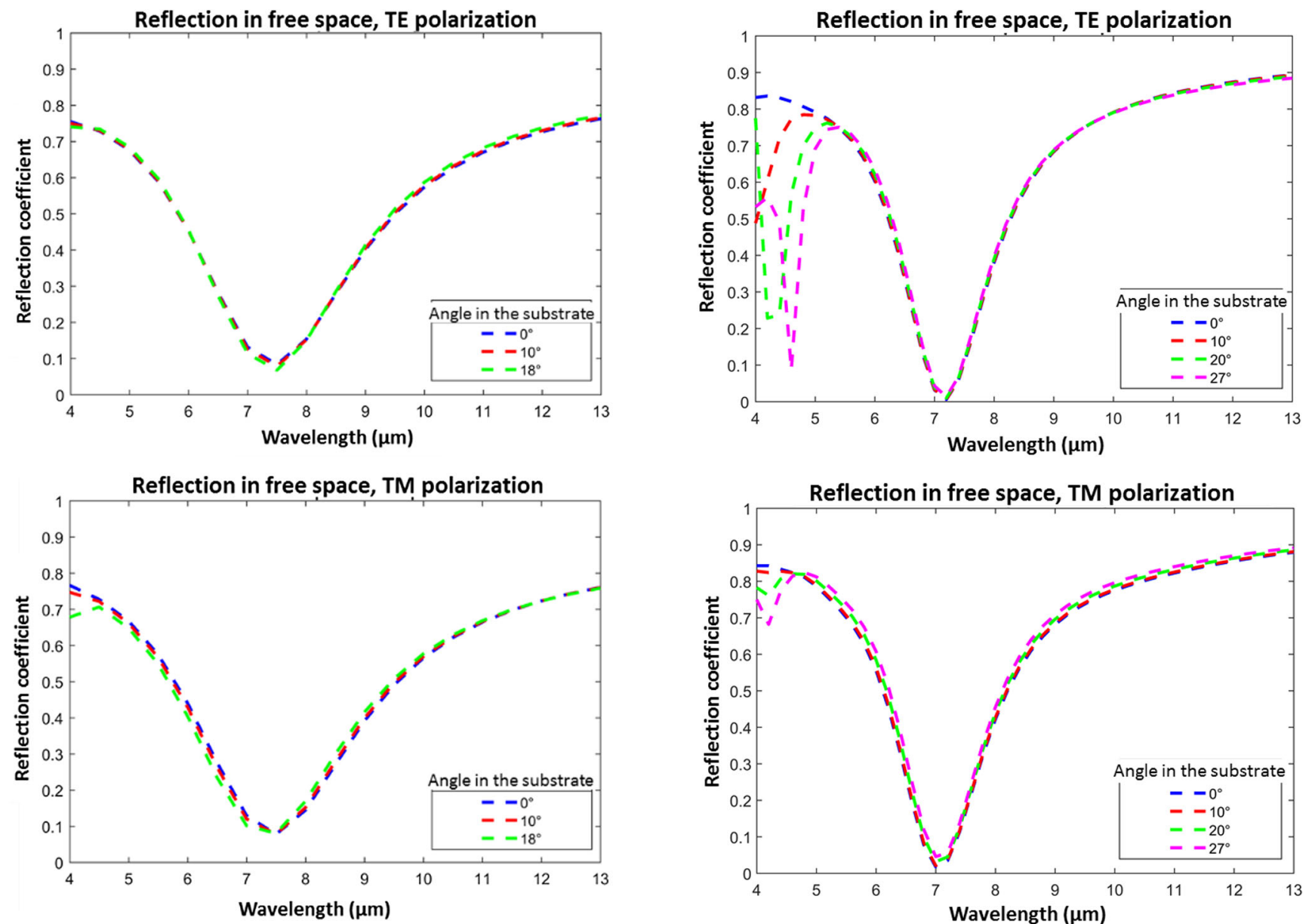

Fig. 14. Reflective spectral bands calculated in TE or TM polarization for several angles of incidence, with a superstrate in $\mathrm{Si}$.

Table 2. Equivalence between Incidence Angle in Air and Incidence Angle in a Superstrate in $\mathrm{ZnS}$

\begin{tabular}{lc}
$\begin{array}{l}\text { Angle of Incidence } \\
\text { in the Air }\end{array}$ & $\begin{array}{c}\text { Angle of Incidence in the ZnS } \\
\text { Superstrate }\end{array}$ \\
\hline $0^{\circ}$ & $0^{\circ}$ \\
$20^{\circ}$ & $8,94^{\circ}$ \\
$40^{\circ}$ & $16,98^{\circ}$ \\
$60^{\circ}$ & $23,18^{\circ}$ \\
$80^{\circ}$ & $26,59^{\circ}$ \\
$90^{\circ}$ & $27,03^{\circ}$ \\
\hline
\end{tabular}

The reflective spectral bands for TE or TM polarization and for different angles of incidence are given in Fig. 15. The central wavelength has a reflection coefficient of $3 \%$ for angles of incidence from $0^{\circ}$ to $27^{\circ}$ in the $\mathrm{ZnS}$ superstrate, which is better than the case with an Si superstrate. The shapes of the reflective spectral bands are the same for all the angles of incidence as well. Moreover, the maximum reflective coefficient is better than the case with the $\mathrm{Si}$ superstrate, with a value of around $85 \%$. Using a smaller index of refraction than Si seems to provide better filtering performance. Finally, the geometries of the plasmonic structures are compatible with UV lithography. Thus, the

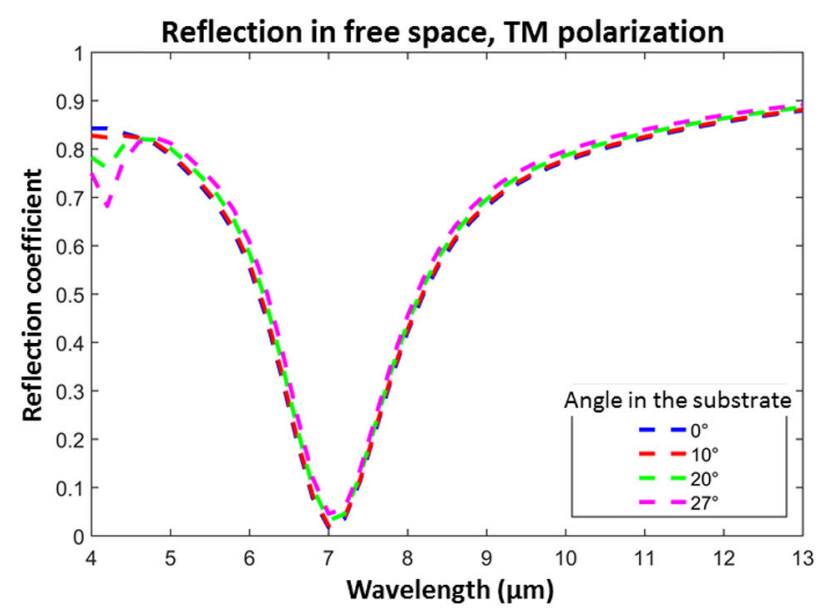

Fig. 15. Reflective spectral bands calculated in TE or TM polarization for several angles of incidence, with $\mathrm{ZnS}$ superstrate.

fabrication of such filters is simpler and less expensive compared to the previous architecture with an Si superstrate.

The study of two materials for immersing the MIM-type plasmonic structures shows that increasing the refractive index of the superstrate drastically improves the filtering performances for rays with a high angle of incidence on the mirrors. A ZnSbased superstrate seems to be a solution to have optimal spectral filtering performance while maintaining plasmonic geometries compatible with UV lithography means. This approach makes a three-block multispectral kaleidoscope-based camera with filters on the mirrors viable. Antireflection coating on the superstrate compatible with high angles of incidence has, however, not been investigated in this study.

\section{PLACING THE FILTER ARRAY ON A PUPIL PLANE}

As mentioned in Section 2, a third strategy to get the kaleidoscope-based camera multispectral is to take advantage of the light field aspect of this camera that transforms the front lens into an array of virtual cameras. Hence, it is possible to place an array of filters at the entrance pupil of the front lens or at a physical stop inside the front lens. In the optimal configuration for a kaleidoscope-based camera presented in Ref. [24], the 


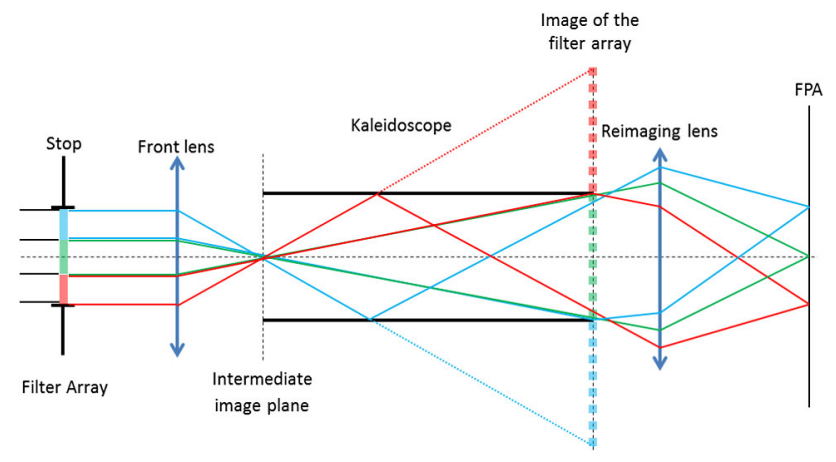

Fig. 16. Principle of a multispectral kaleidoscope-based camera where the array of filters is placed at the entrance pupil of the front lens.

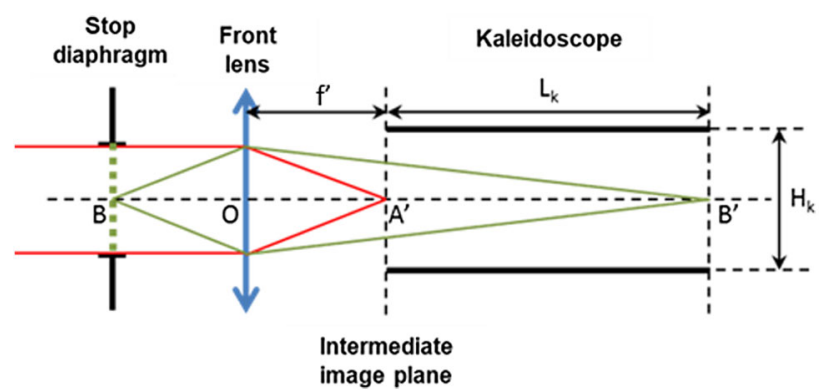

Fig. 17. Layout of a front lens of a kaleidoscope-based system with a stop diaphragm at its entrance pupil and with its exit pupil at the output of the kaleidoscope.

front lens already has a well-defined stop located at the exit of the kaleidoscope. This stop diaphragm is the exit pupil of the front lens. It is, however, possible to add a second stop for the front lens, which could be divided into a multiple subpupils, each subpupil being associated with a subimage, as illustrated in Fig. 16. Placing a different filter in front of each subpupil makes the kaleidoscope-based camera multispectral.

The front lens should conjugate a physical entrance pupil or a stop diaphragm with its physical exit pupil. The pupil conjugation is particularly well known for infrared lenses called reimagers, which conjugate the entrance pupil with the cold stop diaphragm of a cooled detector. However, they are generally composed of two optical stages: an imaging lens and a reimaging lens, separated by an intermediate image plane. For a kaleidoscope-based system and to limit its complexity, the pupil conjugation should be done by a single optical stage that has to provide the dual function of imaging the scene and conjugating the pupils. Therefore, this front lens has to address some optical conditions. We distinguish two cases. The first one, illustrated in Fig. 17, has a stop diaphragm at the entrance pupil of the front lens. The second one, developed in Appendix B, has a stop diaphragm inside the front lens.

We cannot adapt this method to our demonstrator presented in Ref. [25] because the entrance pupil plane is not well defined. Indeed, if we backpropagate the rays from the exit pupil diaphragm (on the exit plane of the kaleidoscope) to the entrance pupil plane in the front lens, we obtain Fig. 18.

We can see that there is a partial overlap between rays belonging to different subimages. This is due to the fact that our

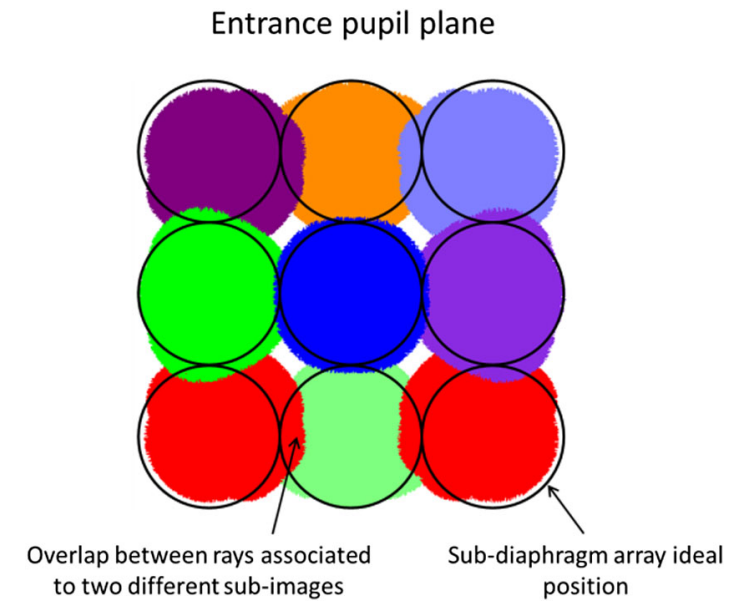

Fig. 18. Image of the subdiaphragms associated with each subimage in the best entrance pupil plane of the demonstrator.

demonstrator does not make a perfect conjugation between the entrance pupil of the front lens and the exit pupil of the front lens. Therefore, this overlapping of rays does not allow the insertion of a filter array without creating a spectral cross talk between subimages.

In the case where a stop diaphragm is positioned at the entrance pupil of the front lens, several optical conditions have to be fulfilled and are deduced hereafter. A first equation links the $F \#$ of the front lens $F \#_{\text {front }}$ with the length of the kaleidoscope $L_{k}$, its section size $H_{k}$, and the number of lateral subimages produced by the kaleidoscope-based camera $N$ [25],

$$
F \#_{\text {front }}=\frac{L_{k}}{N \times H_{k}} .
$$

Taking the approximation $H_{k} \cong \mathrm{FOV} \times f^{\prime}$, FOV being the field of view of the kaleidoscope system, and $f$ the focal length of the front lens, we obtain the following relationship:

$$
L_{k}=F \#_{\text {front }} \times N \times f^{\prime} \times \mathrm{FOV} .
$$

Hence, the dimensions of the kaleidoscope $H_{k}$ and $L_{k}$ can be expressed as a function of the first-order specifications of the front lens $\left(f^{\prime}\right.$ and $\left.F \#_{\text {front }}\right)$ and the camera specifications (FOV and $N$ ). Then we want to determine the position of the entrance pupil with these specifications as well. The distance between the front lens and the stop diaphragm is called $\overline{\mathrm{OB}}$, and the condition for the conjugation of pupils gives the following conjugation relationship:

$$
\frac{1}{f^{\prime}}=\frac{1}{f^{\prime}+L_{k}}-\frac{1}{\overline{\mathrm{OB}}}
$$

This is equivalent to

$$
\overline{\mathrm{OB}}=\frac{-\left(f^{\prime}+L_{k}\right) \times f^{\prime}}{L_{k}} .
$$

Now, by combining Eq. (2) and Eq. (4), we obtain

$$
\overline{\mathrm{OB}}=-\left(\frac{1}{\mathrm{FOV} \times N \times F \#_{\text {front }}}+1\right) \times f^{\prime} .
$$


The stop diaphragm position can thus be expressed as a function of the first-order specifications of the front lens $\left(f^{\prime}\right.$ and $\left.F \#_{\text {front }}\right)$ and the specifications of the camera (FOV, $N$ ).

Now we want to calculate the diameter $\phi_{p}$ of the entrance pupil. In order to have an optimal distribution of the light between the subimages, we know from Ref. [25] that the size of the image of an entrance subpupil conjugated by the front lens must be defined by the output section of the kaleidoscope. So, the size of the image of the entrance pupil, called $\phi_{p}^{\prime}$, is $N$ times larger than the output section of the kaleidoscope. In this case, we obtain the following magnification equation:

$$
\frac{\phi_{p}^{\prime}}{\phi_{p}}=\frac{L_{k}+f^{\prime}}{\overline{\mathrm{OB}}} .
$$

If we express $\phi_{p}^{\prime}$ such that $\phi_{p}^{\prime}=N \times H_{k}$, then the combination of Eq. (2) with Eq. (5) allows us to express the diameter of the stop diaphragm as a function of the kaleidoscope dimensions, the desired number of subimages, and the focal length of the front lens,

$$
\phi_{p}=\frac{f^{\prime} \times N \times H_{k}}{L_{k}} .
$$

We see that the position and the diameter of the entrance pupil of the front lens can be expressed with the specifications of the kaleidoscope-based camera. For the second configuration, where the stop diaphragm is placed inside the front lens, the optical conditions become more complex and a degree of freedom appears. These equations are developed in Appendix B.

Despite the fact that the pupil conjugation can be made by a single optical block, it is, however, difficult to guarantee both a good image quality at the intermediate image plane and a good pupil conjugation. It is then possible to relax the image quality at the intermediate image plane by correcting this degradation with the reimaging lens, but it will be at the cost of increasing the alignment complexity of the two optical blocks and risking vignetting at the borders of the subimages. The quality of the pupil conjugation can be relaxed otherwise, but the diameter of the subpupils should be reduced to avoid spectral cross talks due to the overlap of the subpupils. A third approach to relieve this constraint is to reduce the aperture of the front lens while maintaining the aperture of the overall optical system. To achieve this while maintaining the optimal configuration of a kaleidoscopebased system described in Ref. [25], the focal length of the front lens must be increased, which leads to an increase in the overall system dimension, as illustrated in Fig. 19. Figure 19 describes a configuration of a kaleidoscope-based system where the front lens is opened at $f / 2$ while keeping the same specifications of the kaleidoscope-based system designed in Ref. [25], which is the same field of view and an aperture of $f / 1$. The new system has an overall length of $290 \mathrm{~mm}$, to be compared with the $114 \mathrm{~mm}$ length of the existing kaleidoscope demonstrator with a front lens opened at $f / 1$. In this new design, a stop diaphragm is placed inside the front lens. With each ray dedicated to a subimage having specific colors, we see clearly in Fig. 19 that an array of filters can be placed at the stop diaphragm to get a multispectral kaleidoscope-based system.

In this configuration, there is no spectral cross talk between the subimages. Figure 20 illustrates that the conjugation pupil

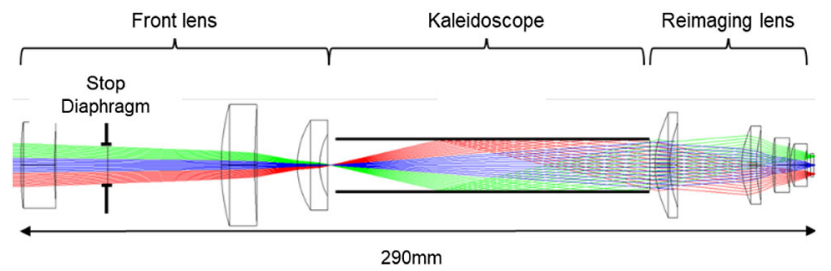

Fig. 19. Kaleidoscope-based architecture where the front lens is opened at $f / 2$ while maintaining the aperture of the whole system to $f / 1$. A stop diaphragm is placed inside the front lens, and an array of filters can be added to get a multispectral kaleidoscope-based system. The rays of the central field point are traced in different colors corresponding to a subimage and a subpupil.

A

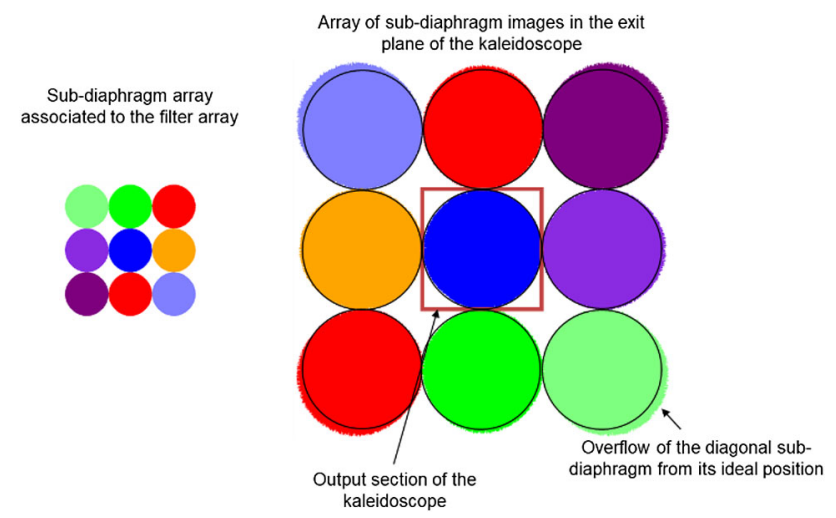

Fig. 20. Illustration of the subpupil conjugation by the front lens of a kaleidoscope-based system. (A) Subpupils at the stop diaphragm; (B) images of the subpupils at the output of the kaleidoscope. Each sub-pupil and its image are associated with a specific color.

is correctly done by the front lens. Figure 20(A) shows the distribution of the light beams in the plane of the filter array, each filter being associated with a specific color and to a circular subdiaphragm. The image of the array of subdiaphragms conjugated by the front lens at the kaleidoscope output is shown in Fig. 20(B). The square output section is framed in brown. The black circles define the ideal subdiaphragm images, and we can see that the diagonal subdiaphragms are slightly overflowing from the circle, but there are no subdiaphragm overlaps. So, the light overflowing from the circles is either vignetted by the circular output diaphragm of the kaleidoscope or forms unwanted subimages, but it does not contribute to spectral cross talk.

Finally, we checked the image quality of the camera. The simulated modulation transfer functions (MTFs) for different field points and for the central subimage, a lateral subimage, and a diagonal subimage are plotted in Fig. 21. It can be seen that the image quality is good with MTFs greater than $20 \%$ at the Nyquist frequency of the detector for all subimages.

Placing an array of filters at a stop diaphragm is a third alternative to get a multispectral kaleidoscope-based camera, provided that the front lens follows some optical conditions. However, the length of this camera can be increased to ensure both a good image quality and no spectral cross talk. Moreover, with this approach, we lose the versatility of a kaleidoscope-based camera 
A
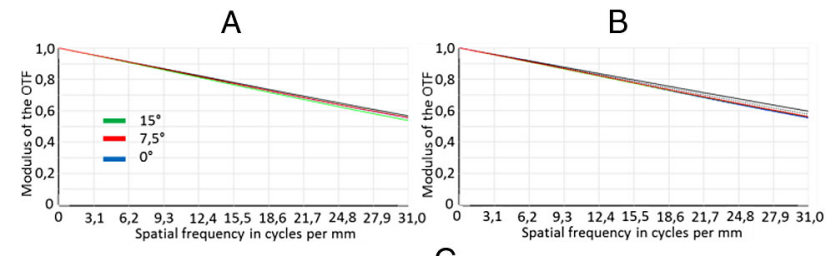

C

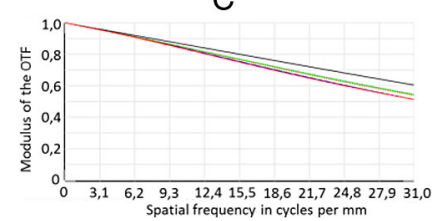

Fig. 21. Simulated MTFs for different field points and for (A) central subimage, (B) lateral subimage, (C) diagonal sub-image. The Nyquist frequency of the detector is $29 \mathrm{cy} / \mathrm{mm}$.

with a generic multispectral block made by the kaleidoscope and the reimaging lens and with the front lens that can be changed to adapt the field of view. Indeed, a specific size of array of filters should be defined for each front lens.

\section{SUMMARY}

Kaleidoscope-based systems have several advantages over other technologies for snapshot multiview infrared imagery. First, they are adapted to the high numerical aperture required by microbolometers while having a great flexibility with the field of view and while limiting the unused pixels between the subimages. They only use conventional components, thus enabling rapid and cost-effective manufacturing and assembling. They also have the advantage of being modular, and the field of view can be adapted by changing only the front lens. However, since it remains a cumbersome camera, it is preferable not to add a fourth optical block. In order to conceive the three-block kaleidoscope-based multispectral camera, three strategies were investigated by placing the filters either on the FPA or on the mirrors or on a pupil plane.

Placing the filters as close as possible in front of the FPA is a straightforward solution that is both simple and effective, but the distance between the filters and the FPA can lead to spectral cross talk between the subimages. The latest technologies for microbolometers, that are WLP or PLP technologies, should be used to limit the amplitude of the spectral cross talk.

Placing filters on the mirrors is the second alternative to having a generic multispectral block made up of the kaleidoscope and the reimaging lens. However, with this solution, the central subimage is not filtered, and notch filters should be used to replace the sets of bandpass and low-pass filters commonly used with microbolometers. In addition, this solution does not allow a high degree of filter flexibility - that is, the ability to easily change the filter array, unlike solutions placing the filters in an image plane or in a pupil plane. Finally, notch filters can be obtained with plasmonic structures, but the high angle of incidence of the rays reflecting on the mirrors degrades the reflective spectral bands of these filters. We demonstrated that immersing the plasmonic structures in a superstrate with a high index of refraction helps to recover an excellent filtering quality.
Finally, placing the filters at the entrance pupil or at a stop diaphragm of the front lens is the third solution to making the kaleidoscope-based camera multispectral. However, the front lens has to validate optical conditions to have a physical entrance pupil or a stop diaphragm and an exit pupil placed at the output of the kaleidoscope. Satisfying both a good image quality and a good pupil conjugation may require increasing the footprint of the kaleidoscope-based camera. Moreover, with this approach, we lose the versatility of a kaleidoscope-based camera with a generic multispectral block and a front lens that can be changed to adapt the field of view.

\section{APPENDIX A: CALCULATION OF THE CROSS TALK OF THE KALEIDOSCOPE-BASED CAMERA}

This Appendix A develops the equations to evaluate the spectral cross talk in the central subimage when the array of filters is separated from the FPA by a window of thickness $e_{\text {window }}$ and by an air thickness $e_{\text {air }}$. To clarify the calculations, we define a frame of reference in Fig. 22 that specifies the $y$ axis and $x$ axis. The central subimage is represented by a blue square with a half-side length $x_{m}$ equal to its half-side width $y_{m}$. Thus, any $(x, y)$ coordinate points of the central subimage are defined as $-x_{m}<x<x_{m}$ and $-y_{m}<y<y_{m}$. Figure 23 shows different positions of the disk area defined by the intersection of the array of filters with a light cone, associated with an image point in the central subimage. The central filter is in blue and is associated with the central subimage, whereas the yellow, green, and red filters are neighboring filters to the central filter. In Fig. 23(A), the surface of the light cone on the array of filters is entirely contained within the central filter, which means that there is no spectral overlap for this image point. In Fig. 23(B), the surface overflows on an adjacent filter. Without any calculations, the extreme case of configuration B occurs when the surface is evenly distributed on the two filters and $50 \%$ of the light is correctly filtered by the central filter. In Fig. 23(C), the surface overflows on two neighboring filters. Finally, in Fig. 23(D), the surface overflows on three neighboring filters. The extreme case of configuration D occurs when the surface is evenly distributed over the four filters and only $25 \%$ of the light is correctly filtered by the central filter.

We first calculate the surface of the intersection of the light cone with the array of filters for any image points of the central subimage. The radius $R$ of the disk surface is linked to the $F \#$ of the system, the cumulative thickness of air between the focal plane and the filters $\left(e_{\text {air }}\right)$, and the thickness of the window $\left(e_{\text {window }}\right)$. The window has an index of refraction of $n$. The path of the light in the window is equivalent to a path of the light in air along a distance of $e_{\text {window }} / n$. The equation giving the radius $R$ is

$$
\begin{aligned}
R & =\left(e_{\text {air }}+\frac{e_{\text {window }}}{n}\right) \times \tan \left(\operatorname{asin}\left(\frac{1}{2 \times F \#}\right)\right) \\
& \approx \frac{e_{\text {air }}+\frac{e_{\text {window }}}{n}}{2 \times F \#} .
\end{aligned}
$$

We now calculate the surface of the intersection of the light cone with the central filter for any image points of the central subimage. To clarify the equations as much as possible, we use a 


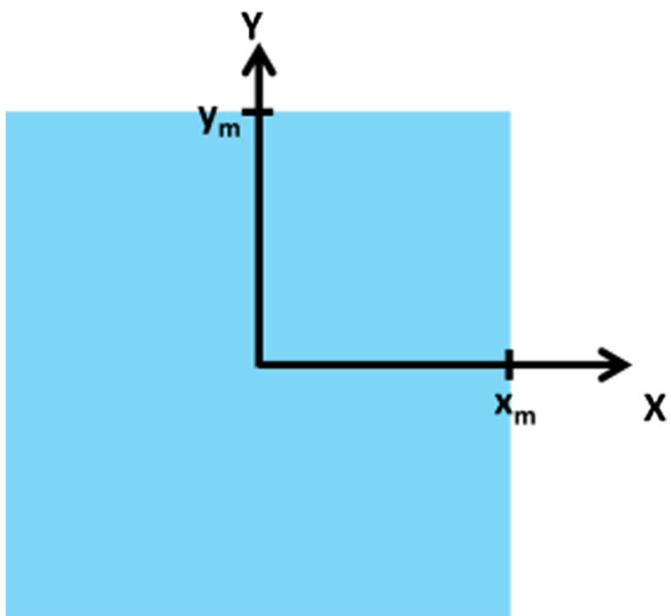

Fig. 22. Frame of reference for spectral cross-talk calculation. The blue square represents the central subimage.

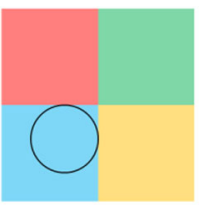

A

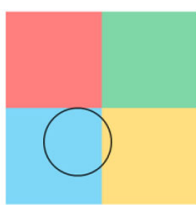

B

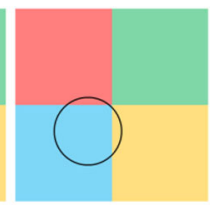

C

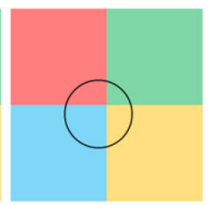

D
Fig. 23. Different possible configurations of the intersection of the array of filters with a light cone associated with an image point in the central subimage.

color code for the different configurations illustrated in Fig. 23. Equations that give the intersection areas on a filter of a color $\mathrm{X}$ will be of the same color.

Configuration A, where the disk does not overlap the adjacent filters, is verified for image points with coordinates $\left(x_{m}, y_{m}\right)$ such as $x_{m}-x>R$ and $y_{m}-y>R$. The equation that gives the surface area of the intersection of the light cone with the central filter is straightforward:

$$
S=\pi \times R^{2} .
$$

Configuration B, where the disk overlaps a single adjacent filter, is verified for image points with coordinates $\left(x_{m}, y_{m}\right)$, such as $y_{m}-y<R$ and $x_{m}-x>R$ (and vice versa). The equation that gives the surface area of the intersection of the light cone with the central filter is

$$
\begin{aligned}
S= & \pi \times R^{2}-\left(R^{2} \times \arccos \left(\frac{y_{m}-y}{R}\right)-\left(y_{m}-y\right)\right. \\
& \left.\times \sqrt{R^{2}-\left(y_{m}-y\right)^{2}}\right) .
\end{aligned}
$$

Configuration C, where the disk overlaps two adjacent filters, is verified for image points with coordinates $\left(x_{m}, y_{m}\right)$, such as $y_{m}-y<R$ and $x_{m}-x<R$. The equation that gives the surface area of the intersection of the light cone with the central filter is

$$
\begin{aligned}
S= & \pi \times R^{2}-\left(R^{2} \times \arccos \left(\frac{y_{m}-y}{R}\right)-\left(y_{m}-y\right)\right. \\
& \times \sqrt{R^{2}-\left(y_{m}-y\right)^{2}}+R^{2} \times \arccos \left(\frac{x_{m}-x}{R}\right) \\
& \left.-\left(x_{m}-x\right) \times \sqrt{R^{2}-\left(x_{m}-x\right)^{2}}\right) .
\end{aligned}
$$

Configuration D, where the disk overlaps three adjacent filters, is verified for image points with coordinates $\left(x_{m}, y_{m}\right)$, such as $\left(y_{m}-y\right)^{2}+\left(x_{m}-x\right)^{2}<R^{2}$. The equation that gives the surface area of the intersection of the light cone with the central filter is

$$
\begin{aligned}
S= & \pi R^{2}-\left(R^{2} \times \arccos \left(\frac{y_{m}-y}{R}\right)-\left(y_{m}-y\right)\right. \\
& \times \sqrt{ }\left(R^{2}-\left(y_{m}-y\right)^{2}\right)+R^{2} \times \arccos \left(\frac{x_{m}-x}{R}\right)-\left(x_{m}-x\right) \\
& \left.\times \sqrt{ }\left(R^{2}-\left(x_{m}-x\right)^{2}\right)\right)+\left(1 / 2 \times R^{2} \times \arccos \left(\frac{y_{m}-y}{R}\right)\right. \\
& -1 / 2 \times\left(y_{-} m-y\right) \times \sqrt{ }\left(R^{2}-\left(y_{m}-y\right)^{2}\right)-1 / 2 \times R^{2} \\
& \times \arccos \left(\frac{\sqrt{R^{2}-\left(x_{m}-x\right)^{2}}}{R}\right)-1 / 2 \times\left(x_{m}-x\right) \\
& \left.\times \sqrt{ }\left(R^{2}-\left(x_{m}-x\right)^{2}\right)+\left(x_{m}-x\right) \times\left(y_{m}-y\right)\right) .
\end{aligned}
$$

Finally, to get the percentage of the amount of correctly filtered light, we simply divide the area of the projected surface on the central filter by the area of the projected surface on the array of filters.

\section{APPENDIX B: DIMENSIONING OF THE FRONT LENS WITH A DIAPHRAGM STOP PLACED BETWEEN TWO OPTICS}

This Appendix B develops the optical conditions for a front lens having both a stop diaphragm and a physical exit pupil at the output of the kaleidoscope. Figure 24 gives a configuration in which the stop diaphragm is placed between two optics of the front lens. The focal length of the optic 1 is denoted $f_{1}^{\prime}$, and the focal length of the optic 2 is denoted $f_{2}^{\prime}$. The focal length of the front lens is denoted $f^{\prime}$. The distance between the optic 2 and the entrance of the kaleidoscope is denoted BFL for back focal length.

As for the configuration developed in Section 4, the firstorder specifications of the front lens are linked with the length of the kaleidoscope $L_{k}$ and the number of lateral subimages $N$ by the following equation:

$$
F \#_{\text {front }}=\frac{1}{\operatorname{FOV}} \frac{L_{k}}{N \times f^{\prime}} .
$$

The focal length $f^{\prime}$ can be derived from the focal lengths of the optic 1 and optic 2 and from their distance thanks to Gullstrand's formula: 


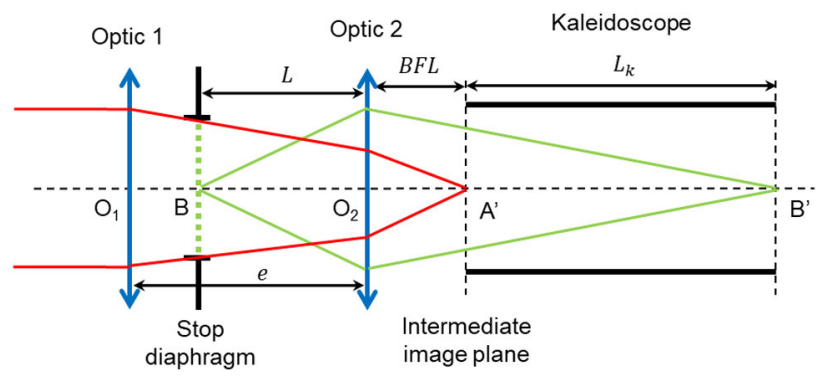

Fig. 24. Configuration of a front lens having a stop diaphragm between two optics and a physical exit pupil at the output of the kaleidoscope.

$$
f^{\prime}=\frac{f_{1}^{\prime} \times f_{2}^{\prime}}{f_{2}^{\prime}+f_{1}^{\prime}-\overline{O_{1} O_{2}}} .
$$

Taking Eq. (B1) and Eq. (B2), we obtain an equation for $\overline{\mathrm{O}_{1} \mathrm{O}_{2}}$

$$
\overline{O_{1} O_{2}}=-f_{1}^{\prime} \times f_{2}^{\prime} \times \frac{\mathrm{FOV}}{H_{k}}+f_{2}^{\prime}+f_{1}^{\prime} .
$$

With the pupil conjugation of the stop diaphragm with the exit pupil of the front lens by the optic 2 , we can find the distance $L$ between the second optic and the stop diaphragm,

$$
L=f_{2}^{\prime} \times \frac{\mathrm{BFL}+L_{k}}{\mathrm{BFL}+L_{k}-f_{2}^{\prime}},
$$

which can be written as

$$
f_{2}^{\prime}=\frac{\mathrm{BFL}+L_{k}}{L+\mathrm{BFL}+L_{k}} L .
$$

With a conjugation relationship on each optic, we obtain

$$
\mathrm{BFL}=\frac{f_{2}^{\prime}\left(f_{1}^{\prime}-e\right)}{\left(f_{1}^{\prime}-e\right)+f_{2}^{\prime}} .
$$

Reference [41] directly gives

$$
f_{1}^{\prime}=\frac{e f^{\prime}}{f^{\prime}-\mathrm{BFL}} .
$$

With Eq. (B7) and the term $e$, we see that this configuration gives some flexibility to position the stop diaphragm. Assuming that optic 2 is a field lens, i.e., $\mathrm{BFL}=0$ and $e=f^{\prime}=\frac{H_{k}}{\mathrm{FOV}}$, the equations become simpler,

$$
f_{1}^{\prime}=\frac{H_{k}}{\mathrm{FOV}}=f^{\prime},
$$

and the pupil conjugation gives a condition for the focal length of the field lens:

$$
\begin{aligned}
& f_{2}^{\prime}=\frac{L \times L_{k}}{L+L_{k}}, \\
& L=\frac{L_{k} \times f_{2}^{\prime}}{L_{k}-f_{2}^{\prime}} .
\end{aligned}
$$

Acknowledgment. We would like to thank the Institut Fresnel for the design of plasmonic filters and SILIOS
Technologies for the manufacture of plasmonic filters. We would like to thank Florence De La Barrière from ONERA for her precious review.

Disclosures. The authors declare no conflicts of interest.

\section{REFERENCES}

1. N. Hagen and M. Kudenov, "Review of snapshot spectral imaging technologies," Opt. Eng. 52, 090901 (2013).

2. https://bertin.fr/sites/default/files/media/bertin/media/brochures/pdf/ Brochure_SecondSight_FR_WEB.pdf.

3. P. D. Atherton, N. K. Reay, J. Ring, and T. R. Hicks, "Tunable FabryPerot filters," Opt. Eng. 20, 206806 (1981).

4. J. Antila, R. Mannila, U. Kantojärvi, C. Holmlund, A. Rissanen, I. Näkki, J. Ollila, and H. Saari, "Spectral imaging device based on a tuneable MEMS Fabry-Perot interferometer," Proc. SPIE 8374, 83740F (2012).

5. N. Gupta, "Hyperspectral imager development at Army Research Laboratory," Proc. SPIE 6940, 69401P (2008).

6. S. Poger and E. Angelopoulou, "Multispectral sensors in computer vision," Technical Report CS 2001-3 (Stevens Institute of Technology, 2001)

7. A. E. Potter, "Multispectral imaging system," U.S. patent $3,702,735$ (14 November 1972).

8. M. R. Descour, "The throughput advantage in imaging Fourier-transform spectrometers," Proc. SPIE 2819, 285-290 (1996).

9. A. R. Harvey, G. Carles, L. Cowan, M. Preciado, J. Ralph, J. Babington, and A. Wood, "The simplicity, complexity, and benefits of multi-aperture imaging in the thermal infrared," Proc. SPIE 10438, 1043806 (2017).

10. Infrared Integrated Systems Ltd., "Multi-array sensor and method of identifying events using the same," E.P. patent 0,973,019," (27 March 2002)

11. B. A. Spiering, "Multispectral imaging system," U.S. patent $5,900,942$ (4 May 1999).

12. Gas Optics Sweden AB, "Quantitative imaging of gas emission utilizing optical techniques," U.S. patent $6,803,577$ “," U.S. patent (28 December 2000).

13. M. N. Abedin, "Multicolor focal plane array detector technology: a review," Proc. SPIE 5152, 279-288 (2003).

14. I. S. Bowen, "The image slicer, a device for reducing loss of light at slit of stellar spectrograph," Astrophys. J. 88, 113-124 (1938).

15. N. S. Kapany, "Fiber optics," in Concepts of Classical Optics, J. Strong, ed. (Dover, 2004), pp. 553-579.

16. G. Courtès, "Méthodes d'observation et étude de l'hydrogène interstellaire en émission," Annales d'Astrophysique 23, 115-244 (1960).

17. P. F. Bernascolle, A. Elichabe, F. Fervel, and J. B. Haumonté, "Standoff CWA imaging system: second sight MS," Proc. SPIE 8358, 83581B (2012).

18. R. Shogenji, Y. Kitamura, K. Yamada, S. Miyatake, and J. Tanida, "Multispectral imaging using compact compound optics," Opt. Express 12, 1643-1655 (2004).

19. A. Portnoy, N. Pitsianis, X. Sun, D. Brady, R. Gibbons, A. Silver, R. Te Kolste, C. Chen, T. Dillon, and D. Prather, "Design and characterization of thin multiple aperture infrared cameras," Appl. Opt. 48, 21152126 (2009).

20. F. de la Barrière, G. Druart, N. Guérineau, and J. Taboury, "Design strategies to simplify and miniaturize imaging systems," Appl. Opt. 50, 943-951 (2011).

21. G. Carles, G. Muyo, N. Bustin, A. Wood, and A. R. Harvey, "Compact multi-aperture imaging with high angular resolution," J. Opt. Soc. Am. A 32, 411-419 (2015).

22. G. Druart, N. Guérineau, R. Haïdar, E. Lambert, M. Tauvy, S. Thétas, S. Rommeluère, J. Primot, and J. Deschamps, "Comparison of a kaleidoscope-based multi-view infrared system with its TOMBO-based counterpart," Proc. SPIE 6992, 69920G (2008).

23. K. Cossu, G. Druart, E. Belhaire, F. Champagnat, and T. Lépine, "Design, implementation and performance analysis of cooled 
infrared cameras with single FPA depth estimation capabilities," Proc. SPIE 9948, 99480B (2016).

24. A. Manakov, J. F. Restrepo, O. Klehm, R. Hegedus, E. Eisemann, $H$. Seidel, and I. Ihrke, "A reconfigurable camera add-on for high dynamic range, multispectral, polarization, and light-field imaging," ACM Trans. Graph. 32, 47 (2013).

25. A. Mas, G. Druart, M. Vaché, S. Favier, A. Alazarine, E. Compain, N. Morin, and N. Guérineau, "Kaleidoscope-based multi-view infrared system," Opt. Lett. 44, 4977-4980 (2019).

26. A. Mas, G. Druart, M. Vaché, S. Favier, A. Alazarine, E. Compain, N. Morin, and N. Guérineau, "Comparison of a kaleidoscope-based multi-view infrared system with its TOMBO-based counterpart," Proc. SPIE 11159, 111590E (2019).

27. S. Pacheco and R. Liang, "Snapshot, reconfigurable multispectral and multi-polarization telecentric imaging system," Opt. Express 22, 16377-16385 (2014)

28. FLIR Systems Inc., "Wafer level packaging of infrared camera detectors," U.S. patent 9,513,172 (6 December 2016).

29. Qualcomm MEMS Technologies, Inc., "Microbolometer supported by glass substrate," U.S. patent 2014/0,267,756A1 (18 September 2014).

30. G. Dumont, W. Rabaud, X. Baillin, J. L. Pornin, L. Carle, V. Goudon, C. Vialle, M. Pellat, and A. Arnaud, "Pixel level packaging for uncooled IRFPA," Proc. SPIE 8012, 80121I (2011).

31. R. Haïdar, G. Vincent, S. Collin, N. Bardou, N. Guérineau, J. Deschamps, and J. L. Pelouard, "Free-standing subwavelength metallic gratings for snapshot multispectral imaging," Appl. Phys. Lett. 96, 221104 (2010).
32. E. Sakat, G. Vincent, P. Ghenuche, N. Bardou, S. Collin, F. Pardo, J. L. Pelouard, and R. Haïdar, "Guided mode resonance in subwavelength metallodielectric free-standing grating for bandpass filtering," Opt. Lett. 36, 3054-3056 (2011).

33. S. Ogawa, K. Okada, N. Fukushima, and M. Kimata, "Wavelength selective uncooled infrared sensor by plasmonics," Appl. Phys. Lett. 100, 021111 (2012).

34. Q. Chen, X. Hu, L. Wen, Y. Yu, and D. R. Cumming, "Nanophotonic image sensors," Small 12, 4922-4935 (2016).

35. S. Ogawa and M. Kimata, "Wavelength-or polarization-selective thermal infrared detectors for multi-color or polarimetric imaging using plasmonics and metamaterials," Materials 10, 493 (2017).

36. P. Bouchon, C. Koechlin, F. Pardo, R. Haïdar, and J. L. Pelouard, "Wideband omnidirectional infrared absorber with a patchwork of plasmonic nanoantennas," Opt. Lett. 37, 1038-1040 (2012).

37. M. Makhsiyan, P. Bouchon, J. Jaeck, J. L. Pelouard, and R. Haïdar, "Shaping the spatial and spectral emissivity at the diffraction limit," Appl. Phys. Lett. 107, 251103 (2015).

38. S. Ogawa and M. Kimata, "Metal-insulator-metal-based plasmonic metamaterial absorbers at visible and infrared wavelengths: a review," Materials 11, 458 (2018).

39. J. P. Hugonin and P. Lalanne, Reticolo Software for Grating Analysis (Institut d'Optique, 2005).

40. R. Lovrincic and A. Pucci, "Infrared optical properties of chromium nanoscale films with a phase transition," Phys. Rev. B 80, 205404 (2009).

41. J. Bentley and C. Olson, Field Guide to Lens Design Vol. FG27 of SPIE Field Guides (SPIE, 2012). 